\title{
UTILIZING MODERN / UPDATED ENERGY SAVING TECHNIQUES TO EXPLOIT BY PRODUCTS FOR BETTER ECONOMY OF SUGAR INDUSTRY
}

\author{
Mohammad Sarfaraz Khan, GM (Plant) \\ Ramzan Sugar Mills, Chiniot
}

\section{INTRODUCTION}

The term Sugar cane byproduct comprises primarily on bagasse, Molasses \& Press mud. However, their contribution would be more or less $30,5 \& 3 \%$ on cane respectively. While, cumulative reflection remains $37-38 \%$.

Principally, byproducts contribute to curtail cost of production to measurable \& even survival extent. Amongst all, bagasse due to their $30 \%$ larger share has greater opportunity is utilized as prime byproduct to reduce cost with energy efficiency.

By \& large, bagasse itself utilize to generate power production on cheapest cost as compared to other sources of fuel. Currently, country - wide sugar industry, utilized bagasse to generate power @ $10-12 \mathrm{KG} / \mathrm{KWH}$ for self-generation. However, Cogeneration can reduce its consumption to 5 $\mathrm{KG} / \mathrm{KWH}$ which is tremendous opportunity for sugar industry to make it proficient (Its Cogen mode steam consumption of extraction - condensing turbines having parameters 110 bar/ 540 Celsius with power output of $31.2 \mathrm{MW}$ consumed steam at $5 \mathrm{Kg} / \mathrm{KWH}$ )

With latest techniques steam consumption can be reduced from conventional 50 to $36-42 \%$ on cane. In order to focus potential opportunities to save bagasse or energy, five distinct areas i.e. a) Mill house Electrification, b) Installation of FFE with integrated vapor distribution, c) capacity utilization, d) Milling equipment \& finally e) Plant automation significantly contributes towards optimization with justified pay back. 
TURBINE DRIVEN MILLING APPLICATIONS

Case study-1

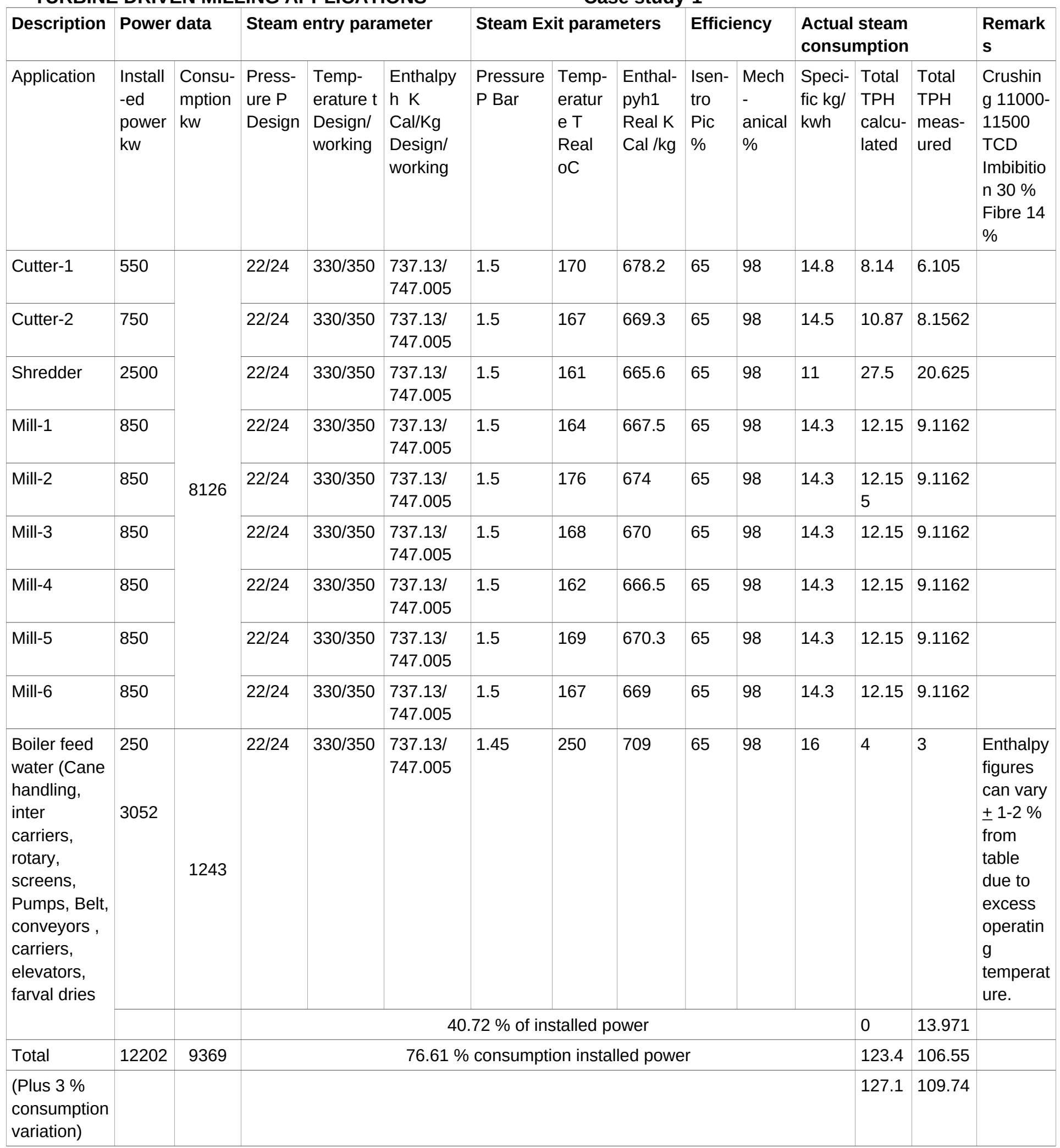


Calculated steam rate basically design rate with respect to unit consumption @ full capacity. However, measured rate@on going rate of crushing which is under capacity crushing.

Isentropic efficiency is basically efficiency inlet \& exit steam pressures. However, friction less adiabatic process is referred as isentropic.

Mechanical efficiency is basically gear box efficiency of transmit power.

MOTOR-VFD DRIVEN MILLING APPLICATIONS UNDER THE SUGAR POWER GENERATION Case study-2

\begin{tabular}{|c|c|c|c|c|c|}
\hline \multirow{2}{*}{$\begin{array}{l}\text { Description } \\
\text { Application }\end{array}$} & \multirow[b]{2}{*}{ Motor I VFD } & \multicolumn{3}{|c|}{ Power Data } & \multirow{2}{*}{\begin{tabular}{|l} 
Remarks \\
Crushing $12000-12500$ TCD \\
Imbibition $27 \%$ Fibre $14 \%$
\end{tabular}} \\
\hline & & $\begin{array}{l}\text { Installed } \\
\text { power kw }\end{array}$ & $\begin{array}{l}\text { VFD Power } \\
\text { KW }\end{array}$ & $\begin{array}{l}\text { Consumption } \\
\text { operating } \\
\text { load KW }\end{array}$ & \\
\hline Cutter-1 & Motor & 1200 & - & 700 & \\
\hline Cutter-2 & Motor & 1000 & - & 800 & \\
\hline Cutter-3 & Motor & 1000 & - & 900 & \\
\hline Shredder (Master) & Motor / VFD & 2500 & $2850 \times 2$ & 1174 & \\
\hline Shredder (Follower) & Motor / VFD & 2500 & $2850 \times 2$ & 1017.29 & \\
\hline Mill-1 & Motor / VFD & 1200 & 1750 & 730 & \\
\hline Mill-2 & Motor / VFD & 1200 & 1750 & 482.68 & \\
\hline Mill-3 & Motor / VFD & 1200 & 1750 & 600.02 & \\
\hline Mill-4 & Motor / VFD & 1200 & 1750 & 499.87 & \\
\hline Mill-5 & Motor / VFD & 1200 & 1750 & 593.66 & \\
\hline Mill-6 & Motor / VFD & 1200 & 1750 & 629.33 & \\
\hline $\begin{array}{l}\text { Mill, shredder, cutters, } \\
\text { Drives }\end{array}$ & & 15400 & 1750 & 8126.85 & \\
\hline $\begin{array}{l}\text { Feeding tables, Inter } \\
\text { carriers, Rotary screens, } \\
\text { Pumps, Belt, Conveyors, } \\
\text { Carriers, Elevators, Farval } \\
\text { drives }\end{array}$ & & 3052 & & 1243 & \\
\hline $\begin{array}{l}\text { Total power for mills } \\
\text { house }\end{array}$ & & 18452 & & 9369.85 & \\
\hline Steam consumption TPH & & & & 103.059 & \\
\hline
\end{tabular}

$5 \mathrm{Kg} / \mathrm{kwh}$ is @110 bar $/ 540 \mathrm{C}$ with $827.35 \mathrm{Kcal} / \mathrm{kg}$. However, actual efficiency attained at turbine where consumption reduce significantly. 
MOTOR-VFD DRIVEN MILLING APPLICATIONS UNDER COGENERATION SCENARIO Case study-3

\begin{tabular}{|c|c|c|c|c|c|}
\hline \multirow{2}{*}{$\begin{array}{l}\text { Description } \\
\text { Application }\end{array}$} & \multirow[b]{2}{*}{ Motor / VFD } & \multicolumn{3}{|c|}{ Power Data } & \multirow{2}{*}{$\begin{array}{l}\text { Remarks } \\
\text { Crushing } 12000-12500 \text { TCD } \\
\text { Imbibition } 27 \% \text { Fibre } 14 \%\end{array}$} \\
\hline & & $\begin{array}{l}\text { Installed } \\
\text { power kw }\end{array}$ & $\begin{array}{l}\text { VFD Power } \\
\text { KW }\end{array}$ & $\begin{array}{l}\text { Consumption } \\
\text { operating } \\
\text { load KW }\end{array}$ & \\
\hline Cutter-1 & Motor & 1200 & - & 700 & \\
\hline Cutter-2 & Motor & 1000 & - & 800 & \\
\hline Cutter-3 & Motor & 1000 & - & 900 & \\
\hline Shredder (Master) & Motor / VFD & 2500 & $2850 \times 2$ & 1174 & \\
\hline Shredder (Follower) & Motor / VFD & 2500 & $2850 \times 2$ & 1017.29 & \\
\hline Mill-1 & Motor / VFD & 1200 & 1750 & 730 & \\
\hline Mill-2 & Motor / VFD & 1200 & 1750 & 482.68 & \\
\hline Mill-3 & Motor / VFD & 1200 & 1750 & 600.02 & \\
\hline Mill-4 & Motor / VFD & 1200 & 1750 & 499.87 & \\
\hline Mill-5 & Motor / VFD & 1200 & 1750 & 593.66 & \\
\hline Mill-6 & Motor / VFD & 1200 & 1750 & 629.33 & \\
\hline $\begin{array}{l}\text { Mill, shredder, cutters, } \\
\text { Drives }\end{array}$ & & 15400 & 1750 & 8126.85 & \\
\hline $\begin{array}{l}\text { Feeding tables, Inter } \\
\text { carriers, Rotary screens, } \\
\text { Pumps, Belt, Conveyors, } \\
\text { Carriers, Elevators, Farval } \\
\text { drives }\end{array}$ & & 3052 & & 1243 & \\
\hline Total power for mills house & & 18452 & & 9369.85 & \\
\hline Steam consumption TPH & & & & 46.84 & Generation@ 0 KG/ \\
\hline
\end{tabular}

\section{SPECIFIC STEAM \% FOR PROCESS sections}

Process applications are classified with $\pm 3-4 \%$ variation at individual plants. While reference base scenario to save energy to produce bagasse as follows;

Juice heating

Evaporators

Vacuum Pans

Miscellaneous

(Pan washing, Centrifugal)

Total

$$
\begin{array}{ll}
= & 8.2 \% \\
= & 20 \% \\
= & 12 \% \\
= & 5 \%
\end{array}
$$

$=\quad 45.2 \%$ on cane 
FALLING FILM EVAPORATORS

FFE is an established feature to bring down steam\% with certain design \& operational advantages,

Short residence time

High heat transfer coefficient Minimum effective temperature difference

Flexibility at capacity fluctuations

Local design \& manufacturing facility
$10-20 \%$ factories have inducted

\section{Automation}

Automation provides the best optimum control of any equipment or process. Comparing to manual control, where performance fluctuates in between two extremes, i.e. optimum best control and worst control due to the various reasons.

Automation facilitates $3-5 \%$ capacity enhancement at milling applications to overcome momentary stoppages

Consistency at process applications stabilize quality and phase changes.

Data control monitoring leads to 4 - $6 \%$ improvement.

Automation controls the equipment or process to highest possible level. A graphical reflection regarding manual and auto control of defecation juice $\mathrm{p.H}$

\section{Process p.H Control Trend graph}

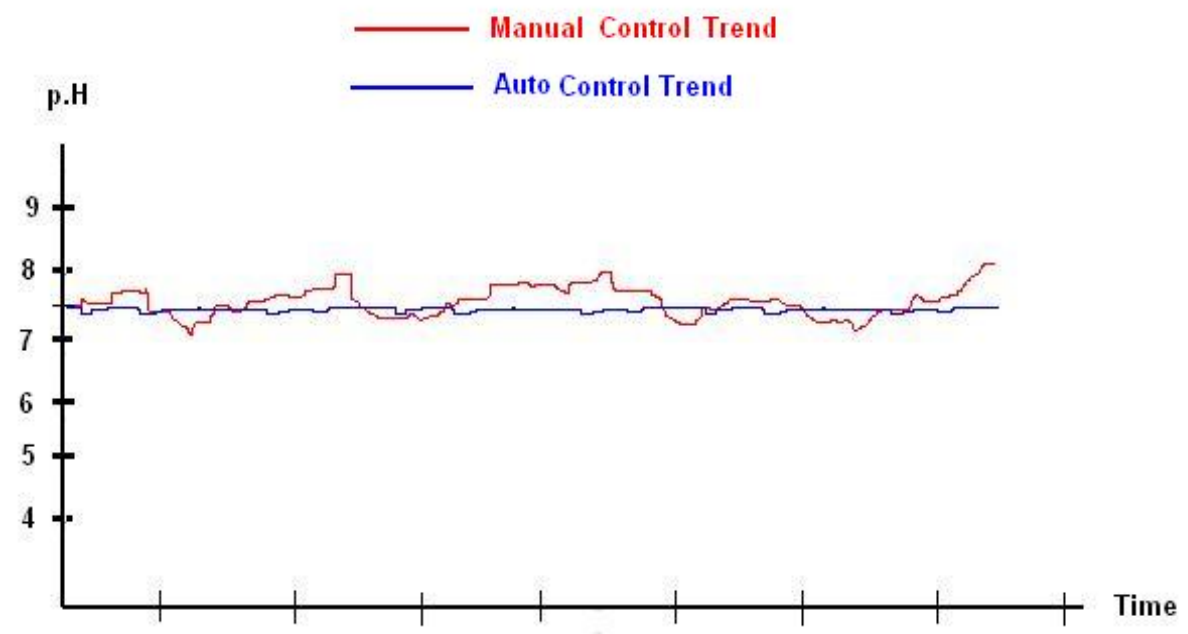




\section{APPLICATION OF 2 ROLLER MILL}

\begin{tabular}{|c|c|c|c|c|c|}
\hline \multicolumn{6}{|c|}{ Comparison of Conventional Mill Units of various configuration operating in Pakistan } \\
\hline \multicolumn{6}{|c|}{ Installed/Absorbed Power calculations based on 8000 TCD crush rate @ 14\% fibre } \\
\hline Mill Type & Conventional & Conventional & Conventional & Conventional & (2 Roller Mill) \\
\hline Pressure Rollers & 3 & 3 & 3 & 3 & 2 \\
\hline $\begin{array}{l}\text { Additional Roller } \\
\text { (Pressure Feeder / } \\
\text { Under Feed) }\end{array}$ & 1 & 2 & 3 & 3 & 1 \\
\hline \multirow[b]{2}{*}{ Unit Configuration } & $\begin{array}{c}\text { Three Roller } \\
\text { with }\end{array}$ & Three Roller with & $\begin{array}{l}\text { Three Roller with } \\
\text { grooved }\end{array}$ & $\begin{array}{l}\text { Three Roller with } \\
\text { grooved }\end{array}$ & \multirow[b]{2}{*}{ 2RM } \\
\hline & under feed & $\begin{array}{l}\text { Toothed } \\
\text { Pressure } \\
\text { Feeders }\end{array}$ & M.D.P.F plus U. F & HD P.F plus U. F & \\
\hline $\begin{array}{l}\text { Installed } \\
\text { Power(KW/TFH) } \\
{ }^{*} \text { Turbine driven) } \\
\star * \text { (Motor-VFD } \\
\text { driven) }\end{array}$ & $18^{*}$ & $20^{*}$ & $22^{*}$ & $23^{*}$ & $14^{\star *}$ \\
\hline $\begin{array}{l}\text { Absorbed Power } \\
\text { (KW/TFH) }\end{array}$ & 13.5 & 15 & 16.5 & 17.25 & 10.93 \\
\hline $\begin{array}{l}\text { Absorbed Power \% } \\
\text { With respect to } \\
\text { (2RM) }\end{array}$ & 19.03 & 27.13 & 33.75 & 36.63 & $\begin{array}{c}\text { Comparison } \\
\text { (Reference } \\
(10.93) \\
\end{array}$ \\
\hline Maintenance Cost & Moderate & High & High & High & Low \\
\hline $\begin{array}{l}\text { First Mill Extraction } \\
\text { (\%) Plain/Reduce } \\
\text { Mittal }\end{array}$ & 71.06/ 74.87 & $72.38 / 75.71$ & 70.31/71.87 & $71.00 / 73.46$ & $74.14 / 77.38$ \\
\hline
\end{tabular}

\section{POWER COMPARISON}

There are two logics of 2 roller mill's induction, enhance crushing @ of reduce power. Incidentally, due to design non-availability of trash plate which consumed on or around 30 $40 \%$ steam consumption in conventional mill. However, low RPM also contributes for more extraction due to larger size mill install at the head of tandem. It's recommended at duty \# 1 rather than center or in last where moisture cannot control due to excess imbibition in most of cases. Higher juice extraction $74 \%$ is ideal reflects in overall extraction around $96 \%$ for tandem.

\section{BASE LINES FOR EFFICIENT STEAM UTILIZATION IN SUGAR PLANTS (ESTIMATIONS)}

These are estimated figures with $5-10 \%$ variations at different sugar plants

Energy Inputs from Bagasse

Energy Inputs from way of condensate return

Energy recovered in steam of total Energy

Contributed by de-superheating water
$91.7 \%$

$8.3 \%$

$64.6 \%$

$0.4 \%$ 
Total heat available in steam from boilers distributed as;

For Process heating, boiling or Prime Movers

Recovered in hot exhaust condensate

Radiation, leakages and Others
$71.8 \%$

$6.9 \%$

$13.3 \%$

$8 \%$

\section{CONCLUSION}

Entire aspects as discussed ultimately reflects the significance of bagasse saving. A crucial byproduct will be going to be a costly commodity in coming years due to switching of multiple conventional sugar mills to Cogen mode. However, availability since November 2015 ranges between Rs. 3000 - 5000/ton shows the rising trend. Therefore, Bagasse as energy fuel can contributes for the future survival of sugar industry.

\section{ACKNOWLEDGMENT}

Author is extremely thankful for the Management of Ramzan Sugar Mills for their support in this study. 